\title{
Intravesical therapy for bladder cancer in the pandemic of Covid-19
}

\author{
Jeremy Y. C. Teoh ${ }^{1} \cdot$ Morgan Roupret $^{2} \cdot$ Shahrokh F. Shariat $^{3,4,5,6,7} \cdot$ Thomas Herrmann $^{8,9}$
}

Received: 6 April 2020 / Accepted: 21 April 2020 / Published online: 30 April 2020

(c) Springer-Verlag GmbH Germany, part of Springer Nature 2020

\section{Dear Editor,}

On 31st December 2019, the World Health Organization (WHO) was informed of cases of pneumonia of unknown etiology detected in Wuhan City, Hubei Province of China. The culprit of the infection was found to be a coronavirus, SARS-CoV-2, and the disease was formally named Covid19. Unfortunately, Covid-19 has spread to more than 200 countries worldwide, and the WHO has officially announced it a pandemic on 11th March 2020 [1].

The exponential increase in the number of Covid-19 cases is accompanied by fear and panic among health care professionals and the general population. Social distancing is recommended to avoid the clustering of people and minimizing the chance of viral transmission; however, this may not be possible for patients who require cancer-related treatments in hospitals.

Thomas Herrmann

thomas.herrmann@stgag.ch

1 Department of Surgery, S.H. Ho Urology Centre, The Chinese University of Hong Kong, Hong Kong, China

2 Sorbonne University, GRC n ${ }^{\circ}$, Predicitive onco-uro, AP-HP, Hôpital Pitié-Salpêtrière, 75013 Paris, France

3 Department of Urology, Medical University of Vienna, Vienna, Austria

4 Institute for Urology and Reproductive Health, I.M. Sechenov First Moscow State Medical University, Moscow, Russia

5 Department of Urology, Weill Cornell Medical College, New York, NY, USA

6 Department of Urology, University of Texas Southwestern, Dallas, TX, USA

7 Department of Urology, Charles University, Prague, Czech Republic

8 Department of Urology, Spital Thurgau AG, Frauenfeld, Switzerland

9 Department of Urology, Hanover Medical School (MHH), Hannover, Germany
In patients with non-muscle-invasive bladder cancer (NMIBC), the use of adjuvant intravesical therapy, either in the form chemotherapy or BCG instillations, has been advocated to optimize disease control. However, it requires repeated hospital visits which may not be ideal in the current pandemic of Covid-19. The mortality rate of Covid-19 increases significantly with age, ranging from $0.32 \%$ in the $20-49$-year age group to $14.8 \%$ in the $\geq 80$-year age group [2]. Bladder cancer tends to occur in chronic smokers with a median age of over 70 years [3], and they are also prone to developing severe sequelae if they happen to contract Covid19. This raises a question on whether we should continue, postpone, or terminate intravesical therapy for NMIBC patients. Polling questions were posted via the \#UroSoMe platform [4], but they did not yield any dominant preferences from the audience possibly due to varying resource availability and changing pandemic situation.

For intravesical chemotherapy, repeated instillations have been shown to improve recurrence rate in intermediate-risk NMIBC. The regimens vary across different centers in terms of number of instillations and duration of treatment course. In a meta-analysis by Sylvester et al. [5], a short intensive schedule within the first 3-4 months versus a longer term (1-year course) but less intensive treatment schedule appears to be equally effective. The results imply that it may be oncologically safe to space out the intravesical chemotherapy instillations, as soon as a longer duration of treatment is planned to be given. However, patients still have to go to the hospital despite a less frequent instillation schedule; the risk of Covid-19 infection must be weighed against the benefit of $38 \%$ reduction in 1-year recurrence rate [6]. Patients who do not opt for intravesical chemotherapy can be followed up with surveillance cystoscopy.

For intravesical BCG therapy, induction course requires 6-weekly instillations, followed by 1-3 years of maintenance course for intermediate- to high-risk NMIBC [7]. Four-tosix weekly instillations are required to achieve a maximum immune-response level which is the rationale behind the induction regimen [8]. At least 1 year of maintenance BCG is required to demonstrate superiority over mitomycin $C$ [9]. 
For patients with high-risk NMIBC, we believe that the benefit of continuing BCG for a better cancer control outweighs the potential risk of Covid-19 infection along the treatment course. To minimize the number of hospital visits, having at least four out of six doses of induction course, and two out of three doses of maintenance course should be considered acceptable. High-risk NMIBC patients who have on-going intravesical BCG therapy for more than 1 year can be safely terminated. For patients with intermediate-risk NMIBC, given the lower risks of disease recurrence and progression, we should consider whether it is still worthwhile to proceed to BCG therapy during this critical time period of Covid-19 pandemic. We can discuss about the option of postponing BCG therapy, but we should emphasize the lack of oncological data in this approach. In countries where there is shortage of BCG, alternative treatments such as device-assisted intravesical chemotherapy can be considered. Countries with whole population BCG vaccination appeared to have a lower incidence and death rate from Covid-19; whether exposure to intravesical BCG has any protective effect towards Covid19 remains to be explored [10].

Avoiding exposure is crucial in preventing Covid-19 infection; however, this may not be possible, especially for patients with high-risk NMIBC. To minimize the risk of Covid-19 infection, patients and health care professionals must be reminded to practice stringent hygienic measures including masking wearing and frequent hand washing in the hospital. Intravesical instillations should be given in single rooms as far as possible; otherwise, adequate distance between patient beds must be ensured. Health care professional should wear personal protective equipment while delivering intravesical therapy.

\section{References}

1. World Health Organization (2020) WHO Director-General's opening remarks at the media briefing on COVID-19 - 11 March 2020. https://www.who.int/dg/speeches/detail/who-director-general-sopening-remarks-at-the-media-briefing-on-covid-19---11-march -2020. Accessed 19 Mar 2020.
2. Oke J, Heneghan C (2020) Global Covid-19 case fatality rates. https://www.cebm.net/global-covid-19-case-fatality-rates/. Accessed 19 Mar 2020.

3. Cumberbatch MGK, Jubber I, Black PC, Esperto F, Figueroa JD, Kamat AM, Kiemeney L, Lotan Y, Pang K, Silverman DT, Znaor A, Catto JWF (2018) Epidemiology of bladder cancer: a systematic review and contemporary update of risk factors in 2018. Eur Urol 74(6):784-795. https://doi.org/10.1016/j.eururo.2018.09.001

4. Gudaru K, Blanco LT, Castellani D, Santamaria HT, Pelayo-Nieto M, Linden-Castro E, Wroclawski ML, Bellote MC, Inarritu JM, da Silva RD, Gauhar V, Adwin Z, Teoh JY (2019) Connecting the urological community : the \#UroSoMe experience. J Endolumin Endourol 2(2):320-e329. https://doi.org/10.22374/jeleu.v2i2.44

5. Sylvester RJ, Oosterlinck W, Witjes JA (2008) The schedule and duration of intravesical chemotherapy in patients with non-muscle-invasive bladder cancer: a systematic review of the published results of randomized clinical trials. Eur Urol 53(4):709-719. https://doi.org/10.1016/j.eururo.2008.01.015

6. Huncharek M, McGarry R, Kupelnick B (2001) Impact of intravesical chemotherapy on recurrence rate of recurrent superficial transitional cell carcinoma of the bladder: results of a meta-analysis. Anticancer Res 21(1B):765-769

7. Oddens J, Brausi M, Sylvester R, Bono A, van de Beek C, van Andel G, Gontero P, Hoeltl W, Turkeri L, Marreaud S, Collette S, Oosterlinck W (2013) Final results of an EORTC-GU cancers group randomized study of maintenance bacillus Calmette-Guerin in intermediate- and high-risk Ta, T1 papillary carcinoma of the urinary bladder: one-third dose versus full dose and 1 year versus 3 years of maintenance. Eur Urol 63(3):462-472. https://doi. org/10.1016/j.eururo.2012.10.039

8. Zlotta AR, van Vooren JP, Huygen K, Drowart A, Decock M, Pirson M, Jurion F, Palfliet K, Denis O, Simon J, Schulman CC (2000) What is the optimal regimen for BCG intravesical therapy? Are six weekly instillations necessary? Eur Urol 37(4):470-477. https://doi.org/10.1159/000020170

9. Bohle A, Bock PR (2004) Intravesical bacille Calmette-Guerin versus mitomycin $\mathrm{C}$ in superficial bladder cancer: formal metaanalysis of comparative studies on tumor progression. Urology 63(4):682-686. https://doi.org/10.1016/j.urology.2003.11.049 (discussion 686-687)

10. Hegarty PK, Kamat A, Zafirakis H, Dinardo A (2020) BCG vaccination may be protective against Covid-19. https://www.resea rchgate.net/publication/340224580_BCG_vaccination_may_be_ protective_against_Covid-19/citations. Accessed 5 Apr 2020.

Publisher's Note Springer Nature remains neutral with regard to jurisdictional claims in published maps and institutional affiliations. 